\title{
S-synchronization and Excitation Constancy in Structural Identifiability Problem of Nonlinear Systems
}

\author{
Nikolay Karabutov ${ }^{1, *}$ \\ ${ }^{1}$ Mirea-Russian Technological University, Department of Problems Control, RU-119454, Moscow, Russia
}

\begin{abstract}
An approach to analysis the structural identifiability (SI) of nonlinear dynamical systems under uncertainty was proposed. S-synchronizability condition of an input is the basis for the structural identifiability estimation of the nonlinear system. A method for obtaining a set containing information about the nonlinear part of the system wasproposed. The decision on SI of the system was based on the analysis of geometric frameworks reflected the state of the system nonlinear part. Geometric frameworks were defined on the specified set. Conditions for structural indistinguishability of geometric frameworks and local identifiability of the nonlinear part were obtained. It shown that a non-S-synchronizing input gives an insignificant geometric framework. This input is a sign of structural non-identifiability of the nonlinear system. The method for estimating the structural identifiability of the nonlinear system was proposed. We show that the structural identifiability is the basis for structural identification of the system. The structural identifiability degree was introduced, and the method of its estimation was proposed.
\end{abstract}

\section{Introduction}

Analysis of recent publications shows that the system identifiability is performed in a parametric space. Many publications are devoted to the study of this problem. Identifiability results are typically presented in the form accepted in the parametric estimation problem. Various methods were proposed for estimating the structural identifiability [1, 2].

Many authors study the parametric identifiability of nonlinear systems (see, e.g. [2-5]). In [2], an approach based on the sensitivity analysis of system output was applied to study identifiability and the analysis of experimental data was used for obtaining parametric identifiability conditions. The critical analysis of approaches applied to the estimate the identifiability of biological models is given in [3]. Models for estimating the identifiability of nonlinear systems are typically based on Taylor series expansion, identifiability tables, and Lie algebra study. Research on practical identifiability was discussed in [4].

The analysis shows that the identifiability of the model is normally understood as the possibility of estimating its parameters and the proposed methods use the nondegeneracy estimation of the information matrix. Similar results were obtained in the parametric estimation theory as the condition of nondegeneracy (completeness of rank) of the matrix input. As a rule, the model structure is set a priori, and, thus, the use of local structural identifiability is not obvious. The concept of structure is widely used in problems of identifiability. The identifiability of a nonlinear system reduces to the parametric identifiability problem and is based on the application of various linearization methods. This extensive field of research does not cover the structural identifiability problem of nonlinear dynamical systems in regarding to deciding on the structure (form, dependence) of the nonlinear system under uncertainty. This statement corresponds to the analysis of structural aspects of the identifiability (identification) system. Also, the question not considered which an input having the excitement constancy property guarantees the structural identifiability of the system. This problem proposed in [6] first.

Also, an important question: "Which input having the excitement constancy property can provide guarantees the structural identifiability of the system" was not considered [6].

In this paper, we study the structural identifiability problem of a nonlinear system. It is important to note that this problem is very complex problem and that methods of formalizing the system structure have not been developed yet. The concept of the structural identifiability ( $h$-identifiability) was introduced in [6]. We propose the approach for solving the structure estimating problem of a nonlinear dynamical system. The approach is based on the analysis of a specific class of frameworks that adequately describe the state of the nonlinear system. The main results obtained in this study can be considered as a generalization of those obtained in $[6,7]$.

\section{Problem statement}

Consider the system

Corresponding author: kn22@yandex.ru 


$$
\begin{aligned}
& \dot{X}=A X+B_{\varphi} \varphi(y)+B_{u} u, \\
& y=C^{T} X,
\end{aligned}
$$

where $u \in R, y \in R$ are the input and output, $\varphi(y)$ is scalar nonlinear function, $B_{u} \in R^{q}, \quad B_{\varphi} \in R^{q}$; $B_{\varphi}=B_{u}=I=[0,0, \ldots, 0,1]^{T}, \quad A \in R^{q \times q} \quad$ is Hurwitz matrix, $C=[1,0, \ldots, 0]^{T}$.

$\varphi(y)$ satisfies the condition

$$
\begin{gathered}
\chi \in F_{\varphi}=\left\{\gamma_{1} \xi^{2} \leq \varphi(\xi) \xi \leq \gamma_{2} \xi^{2}, \xi \neq 0,\right. \\
\left.\varphi(0)=0, \gamma_{1} \geq 0, \gamma_{2}<\infty\right\}
\end{gathered}
$$

The information set

$$
\mathrm{I}_{o}=\left\{u(t), y(t), t \in J=\left[t_{0}, t_{k}\right]\right\}
$$

is known for the system (1).

Problem: use the analysis $\mathrm{I}_{o}$ and estimate the structural identifiability (SI) of the system (1).

\section{Method of design $\mathrm{S}_{\mathrm{ey}}$-framework}

The proposed approach based on the analysis of geometric framework $S_{e y}$. The $S_{e y}$-framework design based on the formation of a set $\mathrm{I}_{N, g}$ contained information about the function $\varphi(y)$ [9]. Apply the differentiation operation to $y(t)$ and denote the obtained variable as $x_{1}$. Accounting $x_{1}$ extends the information the set $I_{o}: I_{e n t}=\left\{I_{o}, x_{1}\right\}$. Accounting $x_{1}$ extends the information the set $\mathrm{I}_{o}: \mathrm{I}_{e n t}=\left\{\mathrm{I}_{o}, x_{1}\right\}$.

Select the subset $\mathrm{I}_{g} \subset \mathrm{I}_{\text {ent }}$ corresponding to the particular solution of the system (5) (steady state). The set $\mathrm{I}_{g}=\mathrm{I}_{\text {ent }} \backslash \mathrm{I}_{t r}$ does not contain data about the transition process in the system Apply the mathematical model

$$
\hat{x}_{1}^{l}(t)=H^{T}[1 u(t) y(t)]^{T}
$$

for obtaining the linear component $x_{1}$. The variable $x_{1}$ is defined on the interval $J_{g}=J \backslash J_{t r}$. Here $H \in R^{3}$ is the vector of model parameters.

Determine the vector $H$ as the solution to the problem

$$
\left.\min _{H} Q(e)\right|_{e=\hat{x}_{1}^{l}-x_{1}} \rightarrow H_{o p t},
$$

where $Q(e)=0.5 e^{2}$.

Find the forecast for variable $x_{1}$ by applying model (4) $\forall t \in \mathrm{I}_{g}$, and generate an error $e(t)=\hat{x}_{1}^{l}(t)-x_{1}(t)$. $e(t)$ depends on the nonlinearity $\varphi(y)$ in the system (1).
So, set $\mathrm{I}_{N, g}=\left\{y(t), e(t) t \in J_{g}\right\}$ has obtained. We apply the designation $y(t)$ supposing $y(t) \in \mathrm{I}_{N, g}$.

Remark 1. Choosing the model structure (4) is the stage for structural identification of the system (1). Simulation results show that the model (4) is applicable in identification systems of objects with static nonlinearity. The model (4) structure choice proposed for another nonlinearity in [9].

The phase portrait $S$ described by function $\Gamma:\{y\} \rightarrow\left\{y^{\prime}\right\}$ does not always guarantee decisionmaking about the nonlinear properties of the system under uncertainty. Therefore, analyze the set $\mathrm{I}_{N, g}$ and go to the space $\mathscr{P}_{y e}=(y, e)$ that we call structural.

Consider the function $\Gamma_{e y}:\{y\} \rightarrow\{e\} \quad \forall t \in J_{g}$ described on the plane $(y, e)$ the change in the framework $S_{e y}$.

Apply the models (4) and present the system (1) as

$$
\begin{aligned}
& S_{y}:\left\{\begin{array}{l}
\dot{\tilde{X}}=A \tilde{X}+I \zeta, \\
\tilde{y}=C^{T} \tilde{X},
\end{array}\right. \\
& S_{\varphi}: e=f\left(y, x_{1}\right),
\end{aligned}
$$

where $\tilde{X} \in R^{q}$ is the variable describing the general solution of the system (1); $\zeta \in R$ is a limited disturbance, which is the result of a procedure applied for the variable definition $e$.

\section{Structural identifiability of system (1)}

Consider the system $S_{\varphi}$ and properties $\mathrm{I}_{N, g}$ that allow solving the problem of structural identification ( $h$ identifiability).

Let the following conditions be satisfied.

B1. The input $u(t)$ is constantly excited at the interval $J$

B2. The input $u(t)$ ensures an informative framework $S_{e y}$.

Definition 1. If $u(t)$ satisfies B1 and B2 conditions, then the input $u(t)$ is representative.

Let the framework $S_{e y}$ closed, and the area $S_{e y}$ is not zero. Denote height $S_{e y}$ as $h\left(S_{e y}\right)$, where $h\left(S_{e y}\right)$ is the distance between two points opposite sides of the framework $S_{e y}$.

Theorem 1. Let (i) the linear part of the system (1) is stable; (ii) the nonlinearity $\varphi(\cdot)$ satisfies the condition (2); (iii) the input $u(t)$ is bounded, and constantly excited; (iv) $h\left(S_{e y}\right) \geq \delta_{S}$, where $\delta_{s}>0$. Then the framework $S_{e y}$ is $h$-identifiable on the set $\mathrm{I}_{N, g}$.

Definition 2. The framework $S_{e y}$ is called $h$ identifiable if theorem 1 holds for $S_{e y}$. 
Let $S_{e y}$ be $h$-identifiable. $h$-identifiability features consider in [6].

A "bad" input that is constantly excited exists. This input gives a so-called "insignificant" $S_{e y}$-framework ( $\mathcal{N S} S_{\text {ey }}$-framework). But the $\mathcal{N} S_{\text {ey }}$-framework can be $h$ identifiable. Identification the system (1) with $\mathcal{N} S_{\text {ey }}$ framework gives results not typical in this case.

Give the conditions for the existence of the framework. Consider a class of nonlinear functions to which the homotopy operation is applies [10]. Let $S_{e y}=F_{S_{e y}}^{l} \cup F_{S_{e y}}^{r}$, where $F_{S_{e y}}^{l}, F_{S_{e y}}^{r}$ are left and right fragments of $S_{e y}$. Define secants for $F_{S_{e y}}^{l}, F_{S_{e y}}^{r}$

$$
\gamma_{S}^{r}=a^{r} y, \gamma_{S}^{l}=a^{l} y,
$$

where $a^{l}, a^{r}$ are estimations obtained least-squares method (LSM).

Theorem 2. Let (i) the framework $S_{e y}$ is $h$ identifiable and has the form $S_{e y}=F_{S_{e y}}^{l} \cup F_{S_{e y}}^{r}$, where $F_{S_{e y}}^{l}, F_{S_{e y}}^{r}$ are the left and right fragments of $S_{e y}$; (ii) secants for $F_{S_{e y}}^{l}, F_{S_{e y}}^{r}$ have forms (6), where $a^{l}, a^{r}$ are LSM-estimations. Then $S_{e y}$ is $\mathcal{N} S_{e y}$-framework if

$$
\left|a^{l}\right|-\left|a^{r}\right|>\delta_{h}, \delta_{h}>0
$$

Remark 2. $\mathcal{N} S_{\text {ey }}$-framework is typical for systems with multiple-valued nonlinearity. Often the framework is the result of inadequate application of an input.

Consider the framework $S_{e y}$. Introduced the designations: $\mathcal{D}_{y}=\operatorname{dom}\left(S_{e y}\right)$ is the definitional domain $S_{e y}, D_{y}=D_{y}\left(\mathcal{D}_{y}\right)=\max _{t} y(t)-\min _{t} y(t)$ is the diameter $\mathcal{D}_{y}$. The set $\mathrm{U}$ contains representative inputs.

Definition 3. If $\mathcal{D}_{y}$ of the framework $S_{e y}$ has the maximum diameter $D_{y}$, then the input S-synchronizes the system (1).

Consider a reference framework $S_{e y}^{r e f} \cdot S_{e y}^{r e f}$ is the framework $S_{e y}$ reflecting all properties of the function $\varphi(y)$. Designate by the diameter $D_{y}\left(S_{e y}^{r e f}\right)$ as $D_{y}^{r e f}$. $D_{y}^{r e f}$ exists if the input the system (1) is Ssynchronizing.

Definitions 2, 3 show if $S_{e y} \cong S_{e y}^{r e f}$, then $\left|D_{y}-D_{y}^{r e f}\right| \leq \varepsilon_{y}$, where $\varepsilon_{y} \geq 0, \cong$ is the proximity sign. Elements of the subset $\mathrm{U}_{\mathrm{S}}$ have property

$$
\left|D_{y}\left(S_{e y}\left(\left.u(t)\right|_{u \in \mathrm{U}_{\mathrm{s}}}\right)\right)-D_{y}^{r e f}\right| \leq \varepsilon_{y} .
$$

We interpret the choice $u_{h}(t) \in \mathrm{U}$ as ensuring synchronization between structures of a model and the system. Therefore, the fulfillment of the condition $d_{h, y}=\max _{u_{h}} D_{y}$ gives $h$-identifiability of the system.

The conditions for $h$-identifiability and for the framework $\mathcal{N} S_{\text {ey }}$ have the form

$$
\begin{gathered}
\left|D_{y}\left(S_{e y}\left(\left.u(t)\right|_{u \in \mathrm{U}_{\mathrm{S}}}\right)\right)-d_{h, y}\right| \leq \varepsilon_{y}, \\
\left|D_{y}\left(S_{e y}\left(\left.u(t)\right|_{u \in \mathrm{UUU}_{\mathrm{S}}}\right)\right)-d_{h, y}\right|>\varepsilon_{y} .
\end{gathered}
$$

Condition (13) can interpreted as the proximity domain

$$
Q_{D}=\left|S_{e y}\left(\left.u(t)\right|_{u \in \mathrm{U}_{\mathrm{s}}}\right)-S_{e y}^{r e f}\right|
$$

which is understood as $\left|\dot{y}(t)-\dot{y}^{r e f}(t)\right| \leq \varepsilon_{y}$ for almost $\forall t \geq t_{0}$.

We will write $\delta Q_{D} \leq \varepsilon_{y}$ if frameworks are close. If the condition $\delta Q_{D} \leq \varepsilon_{y}$ is satisfied for almost $\forall t \geq t^{*}$, then the region $Q_{D}$ call the S-synchronizability region on the set $\left\{u_{h}(t)\right\}$ or the structural identifiability region on the set $\left\{S_{h}\left(u_{h}(t)\right)\right\}$, where $S_{h}$ is the phase portrait of the system (1).

So, we present two criteria (7) and (10) for the existence of the insignificant framework $S_{e y}$. The system $S_{\varphi}$ structure and hence the system (1) is structurally unidentifiable in this case.

Definition 4. If $S_{e y}$ is $h$-identifiable and conditions ||$a^{r}|-| a^{l} \| \leq \delta_{h}$, (8) are satisfied, then the framework $S_{e y}$ or the system (1) is structurally identifiable or $h_{\delta_{h}}$ identifiable.

Definition 4 shows if the system (1) is $h_{\delta_{h}}$-identified, then the framework $S_{e y}$ has the maximum diameter of domain $\mathscr{D}_{y}$.

Let the framework $S$ contain $m$ singularities. We understand features of the function $\varphi(y)$ as loss of continuity on the interval $\mathrm{I}_{y}^{j}$, inflection points or extremum. These features are the sign of the function nonlinearity.

Definition 5. The model (4) is $S M$-identifying if the framework $S_{e y}$ is $h_{\delta_{h}}$-identifiable.

Theorem 3 [8]. Let (i) the input $u(t) \in \mathrm{S}$ is constantly excited; (ii) the system (1) phase portrait have $m$ features; (iii) $S_{e y}$-framework is $h_{\delta_{h}}$-identifiable and contains fragments corresponding to features of the system (1). Then the model (4) is SM -identifying.

Theorem 3 shows if the model (4) is not $S M$ identifying then model (4) structure or the informational set (3) need to change. 
Let $c_{S}$ is the center of the framework $S_{\text {ey }}$ on the set $J_{y}=\{y(t)\}, c_{D_{y}}$ is the center of the domain $\mathscr{D}_{y}$.

Theorem 4. Consider the S-synchronizing set $\mathrm{U}_{\mathrm{s}}$ of the system (1) and let (i) exists $\varepsilon \geq 0$ such that $\left|c_{s}-c_{D_{y}}\right| \leq \varepsilon$; (ii) $\quad\left|a^{l}\right|-\mid a^{r} \| \leq \delta_{h}$, where $a^{l}, a^{r}$ are coefficients of secants for $\left(F_{S_{e y}}^{l}, F_{S_{e y}}^{r}\right) \subset S_{e y}$. Then the system (1) is $h_{\delta_{h}}$-identifiable, and the input $u_{h}(t) \in \mathrm{S}$.

Some subset $\left\{u_{h, i}(t)\right\} \subset \mathrm{U}_{\mathrm{s}} \subseteq \mathrm{U} \quad(i \geq 1)$ which elements have property of S-synchronizability exists. The framework $S_{e y, i}\left(u_{h, i}\right)$ with the diameter $D_{y, i}$ of the domain $\mathscr{D}_{y, i}$ corresponds to every $u_{h, i}(t)$. As $u_{h, i}(t) \in \mathrm{S}$, the diameter $D_{y, i}$ has the property $d_{h, \Sigma}$-optimality.

Definition 6. The framework $S_{e y, i}$ has $d_{h, \Sigma}$ optimality property on the set $\mathrm{U}_{h}$ if $\varepsilon_{\Sigma}>0$ such that $\left|d_{h, \Sigma}-D_{y, i}\right| \leq \varepsilon_{\Sigma} \quad \forall i=\overline{1, \# \mathrm{U}_{h}}$.

Definition 7. If $\left(\left\{u_{h, i}(t)\right\}=\mathrm{U}_{h} \subset \mathrm{U}\right) \&\left(u_{h, i}(t) \in \mathrm{S}\right)$, $i \geq 1$ and frameworks $S_{e y, i}\left(u_{h, i}\right)$ have $d_{h, \Sigma}$-optimality property, then frameworks $S_{e y, i}\left(u_{h, i}\right)$ are structurally indiscernible on sets $\left\{u_{h, i}(t)\right\}, J_{y}\left(u(t)=u_{h, i}(t)\right)$.

Definition 8. If frameworks $S_{e y, i}\left(u_{h, i}\right)$ have $d_{h, \Sigma}$ optimality property, then $S_{e y, i}\left(u_{h, i}\right)$ is locally structurally identifiable on the set $\mathrm{U}_{h}$.

Let the framework $S_{e y, i}\left(u_{h, i}\right)$ having $d_{h, \Sigma}$-optimality property is $S_{e y, i}^{\Sigma}$, and the locally structurally identifiable framework $S_{e y, i}\left(u_{h, i}\right)$ is $S_{e y, i}^{L S I}$.

Therefore, the framework $S_{e y}$ is locally structurally identifiable on the set $\mathrm{U}_{h} \subseteq \mathrm{U}_{\mathrm{S}}$ if

$$
\left(\exists u_{h} \in \mathrm{S}\right) \text {, что }\left(S_{e y} \cong S_{e y}^{\Sigma}\right) \rightarrow S_{e y} \cong S_{e y}^{L S I} .
$$

Remark 3. We have considered the case of symmetric nonlinearity. Therefore, remark and conditions for the existence of the $\mathcal{N S} S_{e y}$-framework are valid. If a nonlinear function does not have the symmetry property, then the further study of this problem is required. Any nonlinearity has features and their use based on the analysis of a prior data.

Definition 9. The framework $S_{e y, i}\left(u_{i} \notin \mathrm{U}_{\mathrm{s}}\right)$ that does not have the $d_{h, \Sigma}$-optimality property is locally structurally unidentifiable.

Remark 4. The described approach applies to the nonlinear system with a dynamic law of the nonlinearity changing, where multi-level structure identifiability analysis used.

The identifiability of the system $S_{y}$ considered in

\section{On constant excitation influence on system identifiability}

In [11], the influence of the excitation constancy (EC) condition on the system identifiability estimation was shown. It is noted that not every input had the ECproperty guarantees the structural identifiability of the system. We present results allowing us to evaluate the effect of this condition.

The property of excitation constancy for input $u(t)$

$$
\operatorname{PE}_{\alpha}: u^{2}(t) \geq \alpha
$$

for $(\exists \alpha>0) \&\left(\forall t \geq t_{0}\right), t \in\left[t_{0}, T\right]$. We will write $u \in \mathbb{P E}_{\alpha}$.

Let the input $u(t) \in \mathscr{P E F} F_{\alpha, \omega_{k}}, \quad u_{k} \in \mathrm{U}_{k}, \mathrm{U}_{k}=\mathrm{U}_{\mathrm{S}}$, where

$$
\begin{gathered}
u_{k}(t):\left(u \in \mathscr{P} E_{\alpha}\right) \&\left(u \in \mathscr{P} F_{\omega_{k}}\right) \&\left(\overline{u_{h} \in \mathrm{S}}\right), \\
\mathcal{P F} F_{\omega_{k}}: u_{k}(t)=R F_{k}\left(\Omega_{k}\right)
\end{gathered}
$$

$\operatorname{RF}\left(\Omega_{k}\right)$ is model based on a Fourier series, $\Omega_{k}=\left\{\omega_{1}, \omega_{2}, \ldots, \omega_{k}\right\} \quad$ is a finite set of frequencies. Therefore, $u_{k} \notin \mathrm{S}$.

We have for $u_{h} \in \mathrm{S}$

$$
\begin{gathered}
u_{h}(t):\left(u_{h} \in \mathcal{P} E_{\alpha}\right) \&\left(u_{h} \in \mathcal{P} F_{\omega_{h}}\right) \&\left(u_{h} \in S\right), \\
\mathcal{P} F_{\omega_{h}}: u_{h}(t)=\mathcal{R F}_{h}\left(\Omega_{h}\right),
\end{gathered}
$$

where $\Omega_{h} \neq \Omega_{k}$.

Obtain from (12), (13)

$$
\left(\mathcal{R F}_{h}\left(\Omega_{h}\right) \neq \mathcal{R F}_{k}\left(\Omega_{k}\right)\right) \Rightarrow S_{e y}^{h} \neq S_{e y}^{k} \Rightarrow S_{e y}^{k}=\mathcal{N} S_{e y} .
$$

and from (14)

$$
\left(\mathcal{D}_{y}\left(S_{e y}^{h}\right) \neq \mathcal{D}_{y}\left(S_{e y}^{k}\right)\right) \Rightarrow\left[D_{y}\left(S_{e y}^{h}\right) \geq D_{y}\left(S_{e y}^{k}\right)\right] .
$$

The domains of the frameworks $S_{\text {ey }}^{h}, S_{\text {ey }}^{k}$ do not coincide, and $S_{e y}^{h}$ is $d_{h, \Sigma}$-optimal on the set $\mathrm{U}_{h}$, then the fulfilment of condition (10) follows from inequality (15). The condition (15) shows that the system (1) nonlinear part structure with the input $u_{k}$ does not coincide with structurally identifiable parameters with $u_{h}$.

Theorem 5. Let 1 ) the input $u_{k}$ satisfy the condition (12); 2) the framework $S_{e y}^{k}$ corresponds to the input $u_{k}$; 3) condition (13) held for $u_{h} \in \mathrm{S}$; 4) conditions (14) and (15) held. Then the $S_{\varphi}$-system is structurally unidentifiable by input $u_{k}$, and structural parameters of the $S_{\varphi}$-system do not correspond to the system (1) with the identifiable framework $S_{e y}^{h}$. [11]. 
Estimate the degree of non-identifiability of the $S_{\varphi}$ system. Let the phase portrait $S$ constructed for the system (1). $S$ and $S_{e y}$-frameworks have the same definitional domains. Therefore, the diameter $D\left(S_{e y}\right)$ of the definitional domain $S_{e y}$ is known. Consider the input set $\left\{u_{i}(t)\right\}$ had the property $\mathcal{P E}_{\alpha}$. Construct framework $S_{e y, i}$ and determine $D_{y, i}\left(S_{e y, i}\right)$ for each $u_{i}(t)$. Let

$$
d_{h, y}=\max _{u_{i}}\left|D_{y}\left(\mathscr{D}\left(S_{e y, i}\right)\right)\right|
$$

and $d_{h, y}$ correspond to the input $u_{h}$. Determine diameters

$$
d_{y, j}=\left|D_{y, j}\left(\mathscr{D}\left[S_{e y, j}\left(u_{j} \in \mathcal{U}\right)\right]\right)\right|
$$

for all inputs $v=\left\{u_{i}(t)\right\} \backslash\left\{u_{h}\right\}$. As $u_{h} \in \mathrm{S}$ then $d_{h, y}>D_{y, j} \forall j \geq 1$. Then the degree of unidentifiable is

$$
S I_{j}=S I\left(S_{e y, j}\right)=\frac{d_{h, y}-d_{y, j}}{d_{h, y}} .
$$

We see that system (1) is structurally identifiable if $S I_{j} \rightarrow 0$. The structural identifiability domain $Q_{D}$ determined by the condition (9).

Remark 5. Select fragments $F_{S}^{l}, F_{S}^{r}$ in the phase portrait $S$. Obtain the non-identifiability degree estimate of the system as

$$
S I=S I(S)=\frac{d_{y}^{l}\left(F_{S}^{l}\right)}{d_{y}^{r}\left(F_{S}^{r}\right)},
$$

where $d_{y}^{l}\left(F_{S}^{l}\right), d_{y}^{r}\left(F_{S}^{r}\right)$ are diameters of the fragments $F_{S}^{l}, F_{S}^{r}$.

The SI of nonlinear systems may depend on the input amplitude. Modify the conditions (12), (13)

$$
\begin{gathered}
u_{k}(t):\left(u \in \mathcal{P E}_{\alpha}\right) \&\left(u \in \mathcal{P} F_{\omega_{k}}\right) \&\left(\overline{u_{h} \in \mathrm{S}}\right), \\
\operatorname{PF}_{\omega_{k}}: u_{k}(t)=\mathcal{R} F_{k}\left(G_{k}, \Omega_{k}\right), \\
u_{h}(t):\left(u_{h} \in \mathcal{P} E_{\alpha}\right) \&\left(u_{h} \in \mathcal{P F} F_{\omega_{h}}\right) \&\left(u_{h} \in \mathrm{S}\right), \\
\mathcal{P F} F_{\omega_{h}}: u_{h}(t)=\mathcal{R} F_{h}\left(G_{h}, \Omega_{h}\right),
\end{gathered}
$$

where $G_{k}, G_{h}$ are parameter vectors of models $R F_{k}$, $\mathrm{RF}_{h}$.

Present the model $R F_{k}$ and $R F_{h}$ in the form

$$
\begin{aligned}
& R F_{h}\left(G_{h}, \Omega_{h}\right)=g_{h} R F_{h}\left(\tilde{G}_{h}, \Omega_{h}\right), \\
& R F_{k}\left(G_{k}, \Omega_{k}\right)=g_{k} R F_{k}\left(\tilde{G}_{k}, \Omega_{k}\right),
\end{aligned}
$$

where $R F_{h}\left(\tilde{G}_{h}, \Omega_{h}\right), \quad R F_{k}\left(\tilde{G}_{k}, \Omega_{k}\right) \quad$ are model modifications (12), (13); $g_{h}=\max _{i} g_{h, i}, i=\overline{1, \# \Omega_{h}}, g_{h, i}$ is element $G_{h} ; g_{k}=\max _{i} g_{k, i}, i=\overline{1, \# \Omega_{k}}$. Designate $g_{p}$ ( $p=k, h$ ) the generalized input amplitude.

The condition (14) transformed into the form

$$
g_{h} R F_{h}\left(\tilde{G}_{h}, \Omega_{h}\right) \neq g_{k} R F_{k}\left(\tilde{G}_{k}, \Omega_{k}\right) .
$$

As the input $u_{h} \in \mathrm{S}$, then $g_{h} \geq g_{k}$. This follows from

$$
\begin{aligned}
& D_{h}\left(S\left(u_{h}\right)\right) \geq D_{k}\left(S\left(u_{k}\right)\right) \Rightarrow \\
& \left|R F_{h}\left(\tilde{G}_{h}, \Omega_{h}\right)\right| \geq\left|R F_{k}\left(\tilde{G}_{k}, \Omega_{k}\right)\right|
\end{aligned}
$$

and the model $R F_{h}\left(\tilde{G}_{h}, \Omega_{h}\right)$ approximates the input ensured S-synchronization of the system (1).

Obtain the $d_{h, \Sigma}$-optimal the diameter $D_{h}\left(s_{e y}^{h}\right)$ from $S\left(u_{h}\right) \Rightarrow S_{e y}^{h}$. As follows from (13), the framework $S_{e y}^{k}$ does not have this property. Hence, $D_{k}\left(S_{e y}^{k}\right)$ gives the input $u_{k} \notin \mathrm{S}$, which has a smaller generalized amplitude.

Theorem 6. Let 1 ) the input $u_{k}$ satisfies the condition (12a); 2) the framework $S_{e y}^{k}$ corresponds to the input $u_{k}$; 3) such input $u_{h} \in \mathrm{S}$ exists that the condition (13a) held; Conditions (14) and (15) held; 4) $g_{h} \geq g_{k}$. Then (i) the $S_{\varphi}$-system is structurally non-identifiable by input $u_{k}$; (ii) structural parameters of the $S_{\varphi}$-system do not correspond to the system (1) with the identified framework $S_{e y}^{h}$.

Example. Consider a system with Bouc-Wen hysteresis (system $S_{B W}$ ) [12]

$$
\begin{gathered}
m \ddot{x}+c \dot{x}+F(x, z, t)=f(t), \\
F(x, z, t)=\alpha k x(t)+(1-\alpha) k d z(t), \\
\dot{z}=d^{-1}\left(a \dot{x}-\beta|\dot{x}||z|^{n} \operatorname{sign}(z)-\gamma \dot{x}|z|^{n}\right),
\end{gathered}
$$

where $m>0$ is weight, $c>0$ is damping, $F(x, z, t)$ is the restoring force, $d>0, n>0, k>0, \alpha \in(0,1), f(t)$ is exciting force, $a, \beta, \gamma$ are some numbers, $y=x$ is the system output. Denote by the system (17) - (19) as $S_{B W}$.

Let $n=1.5, \quad c=2, \quad m=1, \quad \beta=0.5, \quad \alpha=0.7$, $k=0.6, d=a=1$. Set of data have the form $\mathrm{I}_{o}=\left\{u(t), y(t), t \in\left[0 ; t_{e}\right]\right\}, t_{e}>0$. Consider inputs

$$
\begin{gathered}
u_{0}(t)=2-2 \sin (0.15 \pi t), u_{1}(t)=2-2 \sin (0.35 \pi t), \\
u_{2}(t)=2-2 \sin (0.5 \pi t), \\
u_{3}(t)=2-2 \sin (0.15 \pi t)+0.2 \sin (0.35 \pi t) .
\end{gathered}
$$


Design phase portraits $S_{i}$ for the $S_{B W}$-system for each $u_{i}$ and determine the diameters

$$
\begin{aligned}
& D_{y, 0}\left(S_{0}\right)=3.75, D_{y, 1}\left(S_{1}\right)=1.728 \\
& D_{y, 2}\left(S_{2}\right)=1.08, D_{y, 3}\left(S_{3}\right)=3.967
\end{aligned}
$$

The analysis gives $u_{0}(t) \in \mathrm{S}$. Therefore, $S_{0}$ is the reference and $d_{h, y}=D_{y, 0}\left(S_{0}\right)$. Calculate the nonidentifiability degree of the $S_{B W}$-system for various $u_{i}$

$$
S I_{1}=0.549, S I_{2}=0.718, S I_{3}=-0.035 \text {. }
$$

Inputs $u_{1}, u_{2}$ give the structurally unidentifiable $S_{B W}$ system. $S_{B W}$-system with $u_{3}$ is structurally indistinguishable (Fig. 1).

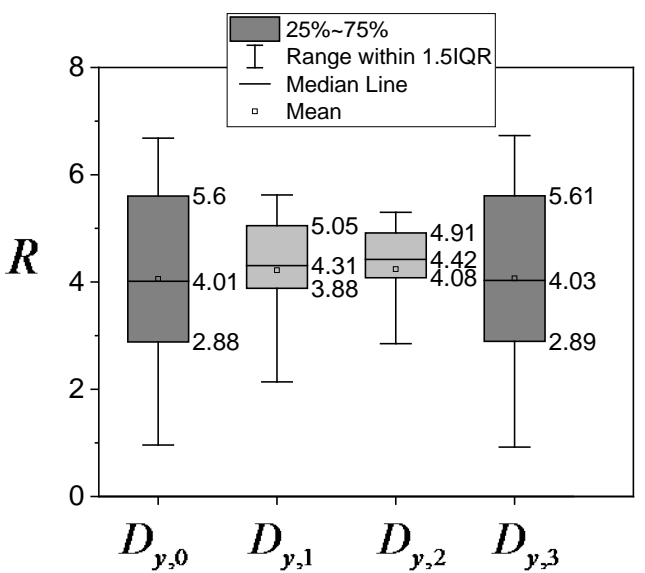

Fig. 1. Diameter $S_{i}$ estimates in the ranked space

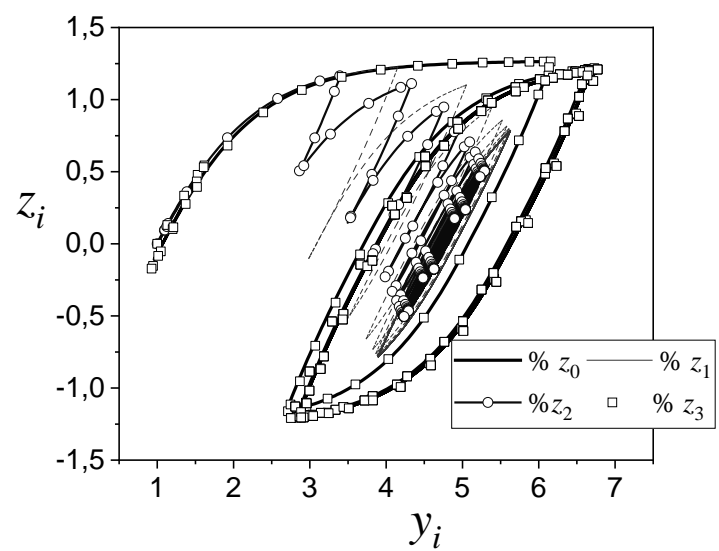

Fig. 2. Hysteresis loops comparison of system with various inputs

Analysis of (22) and Fig. 1 shows that structures $S_{e y, 1}\left(u_{1}\right), S_{e y, 2}\left(u_{2}\right)$ are structures $\mathcal{N} S_{e y}$, and $S_{e y, 3}\left(u_{3}\right)$ structure belongs to class $S_{e y}^{L S I} \cdot R$ is the range of the diameter distribution in Fig. 1

We see that the frequency properties of the input significantly affect the identifiability of the system. It is for the nonlinear part of the system where the change in input properties affects the estimate to structural parameters of the hysteresis. This effect presented in Fig. 2, where the output of the Bouc-Wen model (19) shown for various $u_{i}$.

The identifiability area $Q_{D}$ showed in Fig. 3 for the $S_{B W}$-system. It confirms the made conclusions.

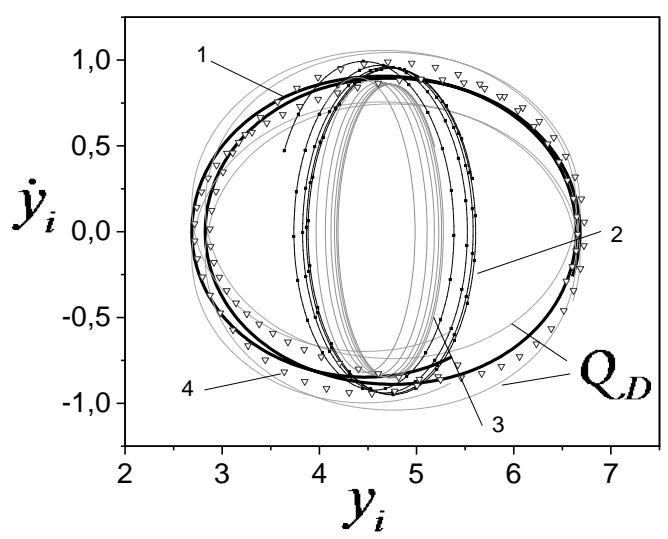

Fig. 3. Area $Q_{D}$ of structural identifiability

So, we propose the method for quantifying estimate the degree identifiability of the system (1). The area of system identifiability obtained.

\section{Conclusion}

The concept of S-synchronizability of the input was introduced. Meeting the input S-synchronizability condition is the basis for structural identifiability and structural identification of the nonlinear system. The input influence on the estimating possibility of the structural parameters nonlinear system has been studied and conditions for the local identifiability and nonidentifiability of the system have been obtained. The identifiability degree the system under uncertainty has been introduced. The influence of the constant excitation of input on the possibility of the system structural identifiability has been studied. The method for calculating the identifiability domain under uncertainty has been proposed.

\section{References}

1. N.A. Balonin Theorems of identifiability (St. Petersburg: Politekhnika publishing house, 2010)

2. J.D. Stigter, R.L. M.Peeters, Proceedings of the ECC 2007 Kos, WeC09.4, 3450-3456 (2007) ISBN: 978-960-89028-5-5

3. O.-T. Chis, J.e R Banga, E. Balsa-Canto, PLOS ONE, 6(4) (2011)

4. M.P. Saccomani, K. Thomaseth, Dynamics of mathematical models in biology. Ed. A. Rogato, V. Zazzu, M. Guarracino (Springer, 2010) ISBN 978-3-319-45722-2 
5. A. Villaverde, A. Barreiro, A. Papachristodoulou, PLOS Computational Biology, 12(10) (2016).

6. N. Karabutov, Global Journal of Science Frontier Research: (A) Physics and Space Science, 18(11) (2018).

7. N. Karabutov, International journal of intelligent systems and applications, 8(7) (2016).

8. N. Karabutov, In Nonlinearity problems, solutions and applications. Volume 1. Ed. L.A. Uvarova, A. B. Nadykto, A.V. Latyshev (New York. Nova Science Publishers, Inc., 2017)

9. N. Karabutov, International journal of intelligent systems and applications, 7(9) (2015)

10. G. Choquet, L'enseignement de la geometrie (Paris: Hermann, 1964)

11. N.N. Karabutov, Mekhatronika, Avtomatizatsiya, Upravlenie, 21(6) (2020)

12. M. Ismail, F. Ikhouane, J. Rodellar, Arch Comput Methods Eng, 16 (2009) 